\title{
Neither Case or Control Status
}

National Cancer Institute

\section{Source}

National Cancer Institute. Neither Case or Control Status. NCI Thesaurus. Code C99273.

The participant is neither a true case or true control for the phenotype under consideration. 